\title{
Some New Results on Weak Integer Additive Set-Labeling of Graphs
}

\author{
N.K. Sudev \\ Department of Mathematics \\ Vidya Academy of Science \& Technology \\ Thrissur-680501, India
}

\author{
K.A. Germina \\ Department of Mathematics \\ University of Botswaana \\ Gaborone, Botswana
}

\begin{abstract}
Let $\mathbb{N}_{0}$ denote the set of all non-negative integers and $\mathcal{P}\left(\mathbb{N}_{0}\right)$ be its power set. An integer additive set-labeling (IASL) of a graph $G$ is an injective function $f: V(G) \rightarrow \mathcal{P}\left(\mathbb{N}_{0}\right)$ such that the induced function $f^{+}: E(G) \rightarrow \mathcal{P}\left(\mathbb{N}_{0}\right)$ is defined by $f^{+}(u v)=$ $f(u)+f(v)$, where $f(u)+f(v)$ is the sumset of $f(u)$ and $f(v)$. An IASL $f$ is said to be an integer additive set-indexer (IASI) if the associated edge-function $f^{+}$is also injective. An IASL $f$ of a given graph $G$ is said to be a weak integer additive set-labeling (WIASL) of $G$ if the cardinality of the set-label of every edge of $G$ is equal to the cardinality of the set-label of at least one end vertex of it. In this paper, we study the admissibility of weak integer additive set-labeling by different graphs.
\end{abstract}

\section{Keywords}

Integer additive set-labeled graphs; weak integer additive setlabeled graphs; sparing number of graphs

\section{Mathematics Subject Classification: 05C78.}

\section{INTRODUCTION}

For all terms and definitions, not defined specifically in this paper, we refer to $([2]),([6])$ and $([16])$. For graph classes, we further refer to ([3]). Unless mentioned otherwise, all graphs considered here are simple, finite and have no isolated vertices.

Let $\mathbb{N}_{0}$ ne the set of all non-negative integers and let $\mathcal{P}\left(\mathbb{N}_{0}\right)$ be its power set. Define the sumset of two non-empty subsets $A$ and $B$ of $\mathbb{N}_{0}$, denoted by $A+B$, is the set $A+B=\{a+b: a \in A, b \in B\}$ (see ([7])). If either $A$ or $B$ is countably infinite, then their sumset is also countably infinite. Hence, all sets we consider in this paper are non-empty finite sets of non-negative integers. We denote the cardinality of a set $A$ by $|A|$. The power set of a set $A$ is denoted by $\mathcal{P}(A)$

Using the terminology of sumsets of two finite sets of non-negative integers, the notion of an integer additive set-labeling has been introduced as follows.

DEFINITION 1. An integer additive set-labeling (IASL) of a graph $G$ is an injective function $f: V(G) \rightarrow \mathcal{P}\left(\mathbb{N}_{0}\right)$ such that the induced function $f^{+}: E(G) \rightarrow \mathcal{P}\left(\mathbb{N}_{0}\right)$ is defined by $f^{+}(u v)=f(u)+f(v)$, where $f(u)+f(v)$ is the sumset of $f(u)$ and $f(v)$. A graph which admits an integer additive labeling is called an integer additive set-labeled graph (IASL-graph). An IASL $f$ is said to be an integer additive set-indexer (IASI) if the associated edge-function $f^{+}$is also injective (see ([4])).

The cardinality of the set-label of an element (a vertex or an edge) of an IASL-graph $G$ is called the set-indexing number of that element. An IASL $f$ of a graph $G$ is said to be a uniform IASL if all the edges of $G$ has the same set-indexing number. If set-indexing number of all edges of an IASL-graph is a positive integer $k$, then $G$ is called a $k$-uniform IASL-graph.

The question whether the set-indexing number of an edge can be equal to the set-indexing number of its end vertices inspired us to establish the following result.

LEMMA 2. ([8]) Let $f: V(G) \rightarrow \mathcal{P}\left(\mathbb{N}_{0}\right)$ be an IASL defined on a graph $G$. Then, for an edge e $=u v$ of $G,\left|f^{+}(u v)\right|=|f(u)|$ if and only if $|f(v)|=1$. More over, $\left|f^{+}(u v)\right|=|f(u)|=f(v) \mid$ if and only if all the three sets are singleton sets.

An edge $e$ of $G$, which has the set-indexing number 1 is called a mono-indexed edge of $G$. Invoking the above notions, we have the following definition.

DEFINITION 3. ([9]) An integer additive set-labeling $f$ of a graph $G$ is said to be a weak integer additive set-labeling (WIASL) of $G$ if the set-indexing number of every edge of $G$ is the same as that of one or all its end vertices. A graph $G$ that admits a weak integer additive set-labeling is called a weak integer additive setlabeled graph (WIASL-graph).

The following results are a necessary and sufficient condition for a graph to admit a WIASL.

LEMMA 4. ([9]) An IASL $f: V(G) \rightarrow \mathcal{P}\left(\mathbb{N}_{0}\right)$ of a given graph $G$ is a WIASL of $G$ if and only if with respect to $f$, at least one end vertex of every edge of $G$ has singleton set-labels.

THEOREM 5. ([9]) A graph G is a WIASL-graph if and only if $G$ is bipartite or it has some edges having singleton set-labels.

In view of the above theorem, we have the following notion.

DEFINITION 6. ([9]) The minimum number of edges having singleton set-labels required for a graph $G$ to admit a WIASL is called the sparing number of $G$, which is denoted by $\varphi(G)$. 


\section{NEW DIRECTIONS}

In this paper, we discuss certain properties and characteristics of certain WIASL graphs and their corresponding sparing numbers. The following result is on the sparing number of an arbitrary graph $G$.

THEOREM 7. Let $G$ be a given WIASL-graph and I be an independent set in $G$ which has highest incidence in $G$. Then, the sparing number of $G$ is the $|E(G-I)|$.

Proof. Let $G$ be a non-bipartite graph which admits a WIASL, say $f$. By Theorem 5 , $G$ has some mono-indexed edges. Also, by Lemma 4 at least one end vertex of every edge of $G$ has a singleton set-label. That is, no two adjacent vertices in $G$ can have nonsingleton set-labels. Therefore, all the vertices of $G$ which have non-singleton set-labels form an independent set in $G$. Let $I$ be an independent set of $G$ having maximal incidence in $G$. Hence, the maximum number of vertices in $G$ which are not mono-indexed is the number of vertices in $I$. An edge, whose one end vertex has a non-singleton set-label, can not have a singleton set-label and hence every edge of $G$ with one end vertex in $I$, does not have a singleton set-label and every edge of $G$ having a non-singleton set-label has one end vertex in $I$. Therefore, all edges in $G-I$ are mono-indexed edges.

An alternate statement to the above problem is as follows.

THEOREM 8. Let $G(V, E)$ be a connected WIASL-graph and let $E_{I}$ be the maximal set of edges of $G$ whose one end vertex is in an independent set of $G$. Then, the sparing number of $G$ is $|E|-$ $\left|E_{I}\right|$.

In the following discussions, we denote an independence set of a graph $G$, which has the highest incidence in $G$ by $I^{*}$. Then, we have the following theorem.

THEOREM 9. If $G(V, E)$ be a non-empty graph and $I^{*}$ be an independent set having maximum incidence in $G$. Then $\varphi(G)=$ $|E|-\sum_{v_{i} \in I^{*}} d\left(v_{i}\right)$.

PROOF. First, recall that number of edges incident on a vertex $v$ in $G$ is the degree of that vertex $d(v)$. Any vertex $v_{i} \in I^{*}$ can have a non-singleton set-label which gives non-singleton set labels to $d\left(v_{i}\right)$ edges incident on it. Since $I^{*}$ is an independent set, the edges thus gain non-singleton set-labels by distinct vertices in $I^{*}$ are all distinct. Therefore, the number of edges having non-singleton sets induced by the vertices in $I^{*}$ is $\sum d\left(v_{i}\right)$. Hence, the number of mono-indexed edges in $G$ is $|E|-\sum_{v_{i} \in I^{*}} d\left(v_{i}\right)$.

If $G$ is an $r$-regular graph, then $\varphi(G)=r \alpha$, where $\alpha$ is the independence number of $G$.

Proof. Let $G$ be an $r$-regular graph. Then, $d(v)=r \forall v \in$ $V(G)$. Hence, any maximal independent set $I$ is an independent set of $G$ with maximal incidence. Then, the number of edges incident on the vertices in $I$ is $\alpha r$, where $\alpha=|I|$, the independence number of $G$. Therefore, by Theorem $9(G)=|E|-\alpha r$.

Figure 1 establishes a WIASL for Petersen Graph $G$. A maximal independent set of the 3-regular graph $G$ is $I=\left\{v_{1}, v_{4}, v_{7}, v_{8}\right\}$. The dotted line lines in $G^{\prime}=G-I$ represent the edges which have one end vertex in $I$. The dotted edges in the corresponding WIASL-graph $G$ represent the mono-indexed edges in $G$.

As the consequences of Theorem 9 and Corollary 2 we immediately have the following remarks.
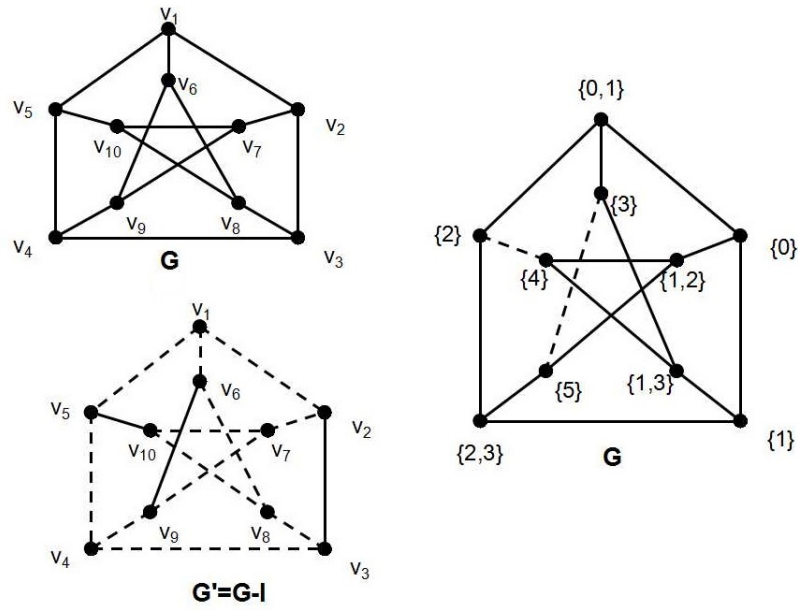

Fig. 1. An illustration to Theorem 7

Let $G$ be a bipartite graph $G$ with bipartition $(X, Y)$. Then, both $|X|$ and $|Y|$ are two independent sets of $G$ such that all edges of $G$ incident on both. Then, the subgraphs $G-X$ and $G-Y$ are edgeless graphs. Therefore, for any bipartite graph $G, \varphi(G)=0$. Let $C_{n}$ be a cycle graph on $n$ vertices. Therefore, the maximal independence set $I$ of $C_{n}$ contains $\left\lfloor\frac{n}{2}\right\rfloor$ vertices. Then, $C_{n}-I$ is edgeless if $n$ is even and has exactly one edge if $n$ is odd. Therefore, $\varphi\left(C_{n}\right)= \begin{cases}0 & \text { if } n \text { is even } \\ 1 & \text { if } n \text { is odd }\end{cases}$

Any independence set of a complete graph $K_{n}$ contains a single vertex of $K_{n}$. Therefore, for any independent set $I=\left\{v_{i}\right\}$ of $K_{n}$, the subgraph $K_{n}-I=K_{n}-\left\{v_{i}\right\}=K_{n-1}$. Hence, $\varphi\left(K_{n}\right)=$ $\left|E\left(K_{n-1}\right)\right|=\frac{(n-1)(n-2)}{2}$.

Next, we consider a particular class of graphs class called split graphs, defined as below. A split graph, denoted by $S(n, r)$, is a graph that can be partitioned in to a clique $K_{n}$ and an independent set $S$ having $r$ vertices. The following theorem establishes the sparing number of a split graph $S(n, r)$.

THEOREM 10. Let $G=S(n, r)$ is a split graph containing a clique $K_{n}$ and an independent $S$ such that $V\left(K_{n}\right)=$ $\left\{u_{1}, u_{2}, \ldots, u_{n}\right\}$ and $S=\left\{v_{1}, v_{2}, \ldots, v_{r}\right\}$. Then,

$$
\varphi(G)= \begin{cases}\left(\begin{array}{c}
n-1 \\
2
\end{array}\right)+\min \{n-1, s\} & \text { if } N(S)=V\left(K_{n}\right) \\
\left(\begin{array}{c}
n-1 \\
2
\end{array}\right) & \text { if } N(S) \subsetneq V\left(K_{n}\right)\end{cases}
$$

where $s=\min \left\{\sum_{u_{i} \sim v_{i}}\left(d_{S}\left(v_{j}\right)-1\right)\right\}$, for all $1 \leq i \leq n$.

Proof. Let $G=S(n, r)$. If $N(S) \subsetneq V\left(K_{n}\right)$, then there exist some vertices of $K_{n}$ which are not adjacent to any vertex in $S$. without loss of generality, let $v_{1}$ be a vertex of $K_{n}$ which is not adjacent to any vertex in $S$. Since only one vertex in $K_{n}$ can be independent, $I=s \cup\left\{v_{1}\right\}$ is a maximal independent set in $G$. Then, $G-I=K_{n}-v_{1}=K_{n-1}$. Then, by Theorem $7 \varphi(G)=$ $\left|E\left(K_{n-1}\right)\right|=\frac{(n-1)(n-2)}{2}$.

If $N(S)=V\left(K_{n}\right)$, then every vertex of the clique $K_{n}$ of $G$ is adjacent to some vertex of the independent set $S$. Then, the maximal independent set $I$ in $G$ containing the end vertices of maximum edges of $G$ contains at most one vertex of $K_{n}$. If a vertex $u_{i}$ of $K_{n}$ belongs to $I$, then the vertices of $S$ that are adja- 
cent to $u_{i}$ will become mono-indexed and the edges incident one these vertices, other than those incident on $u_{i}$ also become monoindexed. The number of such newly mono-indexed edges will be $s=\min \left\{\min \left\{\sum_{u_{i} \sim v_{i}}\left(d_{S}\left(v_{j}\right)-1\right)\right\}\right.$. Therefore, the minimum number of mono-indexed edges in $G$ is $\left(\begin{array}{c}n-1 \\ 2\end{array}\right)+\min \{n-1, s\}$. This completes the proof

Figure 2 illustrates the two cases mentioned in the above theorem.
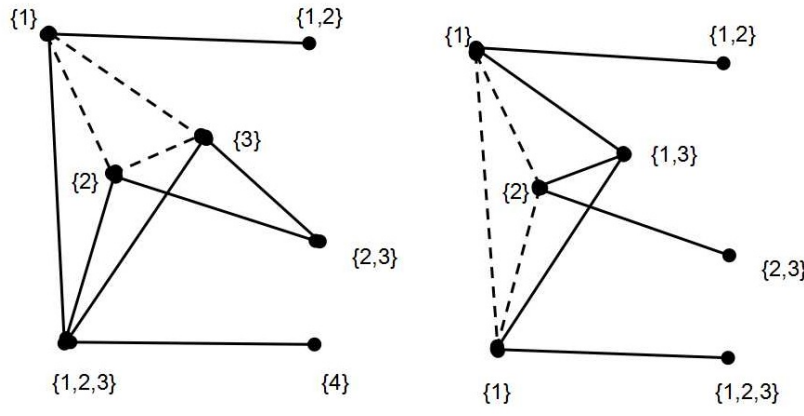

Fig. 2. WIASLs of two types of Split Graphs

In view of Theorem 7, we shall now determine the sparing number of certain graph operations.

The union of two graphs $G_{1}\left(V_{1}, E_{1}\right)$ and $G_{2}\left(V_{2}, E_{2}\right)$, denoted by $G_{1} \cup G_{2}$, is the graph with vertex set $V_{1} \cup V_{2}$ and the edge set $E_{1} \cup E_{2}$. The sparing number of graph unions have been determined in [9]. We provide a better alternate proof for the following theorem on the sparing number of the union of two graphs using Theorem 7

THEOREM 11. Let $G_{1}$ and $G_{2}$ be two graphs and let $G=$ $G_{1} \cup G 2$. Then, $\varphi\left(G_{1} \cup G_{2}\right)=\varphi\left(G_{1}\right)+\varphi\left(G_{2}\right)-\varphi\left(G_{1} \cap G_{2}\right)$. If $G_{1}$ and $G_{2}$ are edge-disjoint graphs, then $\varphi\left(G_{1} \cup G_{2}\right)=$ $\varphi\left(G_{1}\right)+\varphi\left(G_{2}\right)$.

Proof. Let $I_{1}$ and $I_{2}$ be the maximal independence sets in $G_{1}$ and $G_{2}$ respectively. Then, $I=I_{1} \cup I_{2}$ is a maximal independent set of $G=G_{1} \cup G_{2}$. Now, the set of edges of $G$ with one end vertex in $I$ is $E_{I}=E_{I_{1} \cup I_{2}}=E_{I_{1}} \cup E_{I_{2}}$. Then, by Theorem 8, we have

$$
\begin{aligned}
& \varphi(G)=|E(G)|-\left|E_{I}\right| \\
&=\left|E\left(G_{1} \cup G_{2}\right)\right|-\left|E_{I_{1}} \cup E_{I_{2}}\right| \\
&= {\left[\left|E\left(G_{1}\right)\right|+\left|E\left(G_{2}\right)\right|-\left|E\left(G_{1} \cap G_{2}\right)\right|\right] } \\
&-\left[\left|E_{I_{1}}\right|+\left|E_{I_{2}}\right|-\left|E_{I_{1}} \cap E_{I_{2}}\right|\right] \\
&= {\left[\left|E\left(G_{1}\right)\right|-\left|E_{I_{1}}\right|\right]+\left[\left|E\left(G_{2}\right)\right|-\left|E_{I_{2}}\right|\right] } \\
&-\left[\left|E\left(G_{1} \cap G_{2}\right)\right|-\left|E_{I_{1}} \cap E_{I_{2}}\right|\right] \\
&=\varphi\left(G_{1}\right)+\varphi\left(G_{2}\right)-\varphi\left(G_{1} \cap G_{2}\right) .
\end{aligned}
$$

If $G_{1}$ and $G_{2}$ are edge-disjoint graphs, then $G_{1} \cap G_{2}$ is an edgeless graph. Then, $E_{I_{1}} \cup E_{I_{2}}$ is a null set. Therefore, we have $\varphi(G)=$ $\varphi\left(G_{1}\right)+\varphi\left(G_{2}\right)$.

The admissibility of WIASLs by graph join has also been studied in ([9]) and have established the following theorem, using the fact that every vertex of $G_{1}$ is adjacent to all vertices of $G_{2}$.
THEOREM 12. ([9]) The join of two WIASL-graphs $G_{1}$ and $G_{2}$ admits a WIASL if and only if either $G_{1}$ or $G_{2}$ is 1-uniform.

In view of the above theorem, we now determine the sparing number of the join two given graphs in the following theorem.

THEOREM 13. The sparing number of the join of two arbitrary graphs $G_{1}\left(V_{1}, E_{1}\right)$ and $G_{2}\left(V_{2}, E_{2}\right)$ is $\min \left\{\varphi\left(G_{1}\right)+\left|E_{2}\right|\left(\beta_{1}+\right.\right.$ $\left.1), \varphi\left(G_{2}\right)+\left|E_{1}\right|\left(\beta_{2}+1\right)\right\}$, where $\beta_{1}, \beta_{2}$ are the covering numbers of $G_{1}$ and $G_{2}$ respectively.

PROOF. For any graph $G$ which admits a WIASL, by Theorem 7. the maximum number of vertices that are not mono-indexed is $\alpha$, where $\alpha$ is the independence number of $G$. Then, the minimum number of mono-indexed vertices in the graph $G$ is $|V(G)|-\alpha=$ $\beta$, the covering number of $G$.

Now, let the graph join $G_{1}+G_{2}$ admits a WIASL. Then, by Theorem 12 either $G_{1}$ or $G_{2}$ is 1-uniform. Hence, we proceed by considering the following cases.

Case-1: Let $G_{1}$ be 1-uniform. Then, all edges of $G_{1}$ are monoindexed. As stated earlier, the number of mono-indexed vertices in $G_{2}$ is $\beta_{2}$, its covering number. Since each vertex of $G_{1}$ is adjacent all vertices of $G_{2}$, the number of mono-indexed vertices between $G_{1}$ and $G_{2}$ is $\beta_{2}\left|E_{2}\right|$. More over, the number of mono-indexed edges in $G_{2}$ is $\varphi\left(G_{2}\right)$. Therefore, total number of mono-indexed edges in this case is $\left|E_{1}\right|+\beta_{2} .\left|E_{1}\right|+\varphi\left(G_{2}\right)$.

Case-2: Let $G_{2}$ be 1-uniform. Then, as explained in Case-1, the number of mono-indexed edges in $G_{1}+G_{2}$ is $\left|E_{2}\right|+\beta_{1}$. $\left|E_{2}\right|+$ $\varphi\left(G_{1}\right)$.

Therefore, the sparing number of $G_{1}+G_{2}$ is $\min \left\{\varphi\left(G_{1}\right)+\left(\beta_{1}+\right.\right.$ 1) $\left.\left|E_{2}\right|, \varphi\left(G_{2}\right)+\left(\beta_{2}+1\right)\left|E_{1}\right|\right\}$.

Figure 3 illustrates the above theorem on the sparing number of graph joins.
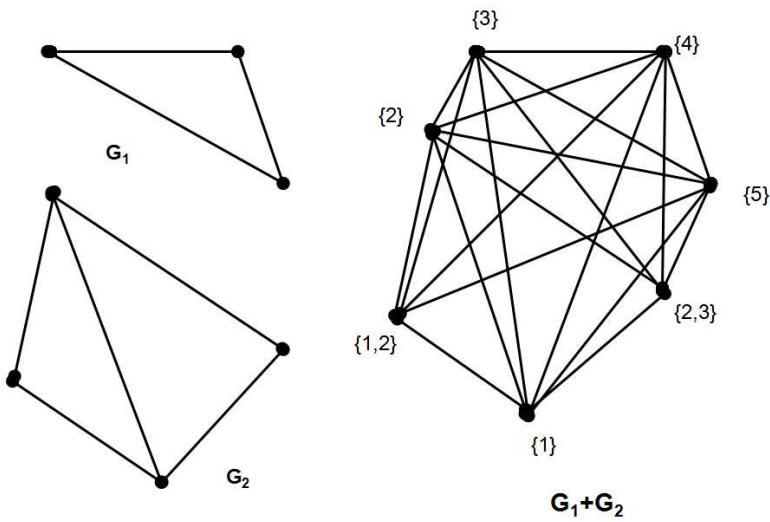

Fig. 3. A WIASL for the graph join $G_{1}+G_{2}$.

In view of Theorem 7 an alternate method to determine the sparing number of the join of two arbitrary graphs is established in the following theorem. 
THEOREM 14. Let $G_{1}\left(V_{1}, E_{2}\right)$ and $G_{2}\left(V_{2}, E_{2}\right)$ be two graphs with independence numbers $\alpha_{1}$ and $\alpha_{2}$ respectively. Then, the sparing number of the graph join $G_{1}+G_{2}$ is is $\left|E_{1}\right|+\left|E_{2}\right|+\left|V_{1}\right|\left|V_{2}\right|-$ $\max \left\{\left(\left|V_{2}\right|\left(\left|I_{1}\right|-1\right)+\left|E_{I_{1}}\right|\right),\left(\left|V_{1}\right|\left(\left|I_{2}\right|-1\right)+\left|E_{I_{2}}\right|\right)\right\}$, where, for $j=1,2, I_{j}$ is an independence set of the graph $G_{j}$ with maximal incidence in $G_{j}$ and $E_{I_{j}}$ is the set of edges in $G_{1}+G_{2}$ which have one end vertex in $I_{j}$.

PROOF. Consider two arbitrary graphs $G_{1}\left(V_{1}, E_{2}\right)$ and $G_{2}\left(V_{2}, E_{2}\right)$. Note that the number of edges in $G_{1}+G_{2}$ is $\left|E_{1}\right|+$ $\left|E_{2}\right|+\left|V_{1}\right|\left|V_{2}\right|$. Let $I_{1}$ be an independence set of $G_{1}$ with maximal incidence in $G_{1}$ and $I_{2}$ be an independence set of $G_{2}$ with maximal incidence in $G_{2}$. If $I_{1}$ has more incidences than $\left|I_{2}\right|$, then the maximum number of edges having non-singleton set-label in $G_{1}+G_{2}$ is $\left|E_{2}\right|+\left|I_{1}\right|\left|V_{2}\right|+\sum_{v_{i} \in I_{1}} d\left(v_{i}\right)$. Hence, in this case, the number of mono-indexed edges in $G_{1}+G_{2}$ is $\left|E_{1}\right|+\left|E_{2}\right|+\left|V_{1}\right|\left|V_{2}\right|-\left[\left|E_{2}\right|+\right.$ $\left.\left|I_{1}\right|\left|V_{2}\right|+\sum_{v_{i} \in I_{1}} d\left(v_{i}\right)\right]=\left|E_{1}\right|+\left|V_{1}\right|\left|V_{2}\right|-\left|I_{1}\right|\left|V_{2}\right|-\sum_{v_{i} \in I_{1}} d\left(v_{i}\right)$. Similarly, if $I_{2}$ has more incidences than $I_{1}$, then the number of mono-indexed edges in $G_{1}+G_{2}$ in this case is $\left|E_{2}\right|+\left|V_{1}\right|\left|V_{2}\right|-$ $\left|I_{2}\right|\left|V_{1}\right|-\sum_{u_{j} \in I_{2}} d\left(u_{j}\right)$. Therefore, the sparing number of $G_{1}+G_{2}$ is $\left|V_{1}\right|\left|V_{2}\right|+\min \left\{\left(\left|E_{1}\right|-\left|I_{1}\right|\left|V_{2}\right|-\sum_{v_{i} \in I_{1}} d\left(v_{i}\right)\right),\left(\left|E_{2}\right|-\left|I_{2}\right|\left|V_{1}\right|-\right.\right.$ $\left.\left.\sum_{u_{j} \in I_{2}} d\left(u_{j}\right)\right)\right\}$

The next theorem is on the sparing number of the join of two given regular graphs.

THEOREM 15. Let $G_{1}$ be an r-regular graph of order $n_{1}$ and $G_{2}$ be an s-regular graph of order $n_{2}$. Then, the sparing number of the graph join $G_{1}+G_{2}$ is $\frac{1}{2}\left[\min \left\{\left(n_{1}\left(r+2 \beta_{2}\right)+s\left(\beta_{2}-\right.\right.\right.\right.$ $\left.\left.\left.\left.\alpha_{2}\right)\right),\left(n_{1}\left(s+2 \beta_{1}\right)+r\left(\beta_{1}-\alpha_{1}\right)\right)\right\}\right]$.

Proof. Let $G_{1}$ be an $r$-regular graph of order $n_{1}$ and $G_{2}$ be an $s$-regular graph of order $n_{2}$. Then, the total number of edges in $G_{1}$ and $G_{2}$ are $\frac{1}{2} r n_{1}$ and $\frac{1}{2} s n_{2}$ respectively. Let $I_{1}$ and $I_{2}$ be two independent sets, respectively in $G_{1}$ and $G_{2}$ with maximum incidences and let $\alpha_{1}$ and $\alpha_{2}$ be the independence numbers of $G_{1}$ and $G_{2}$ respectively. Then, $\left|I_{1}\right|=\alpha_{1}$ and $\left|I_{2}\right|=\alpha_{2}$. Therefore, the maximum number of vertices that can have non-singleton set-labels in $G_{1}$ and $G_{2}$ are $\alpha_{1} r$ and $\alpha_{2} s$ respectively.

If $\alpha_{1} r \leq \alpha_{2} s$, then $\alpha_{2}$ is the number of vertices in $G_{1}+G_{2}$ having non-singleton set-labels. Therefore, the number of edges having non-singleton set-labels in $G_{1}+G_{2}$ is $\alpha_{2}\left(s+n_{1}\right)$. Hence, the number of mono-indexed vertices in $G_{1}+G_{2}$ here is $\frac{1}{2}\left(r n_{1}+s n_{2}+\right.$ $\left.n_{1} n_{2}\right)-\alpha_{2}\left(s+n_{1}\right)=\frac{1}{2}\left[n_{1}\left(r+2 n_{2}-2 \alpha_{2}\right)+s\left(n_{2}-2 \alpha_{2}\right)\right]=$ $\frac{1}{2}\left[n_{1}\left(r+2 \beta_{2}\right)+s\left(\beta_{2}-\alpha_{2}\right)\right]$. Similarly, if $\alpha_{1} r \geq \alpha_{2} s$, then the number of mono-indexed vertices in $G_{1}+G_{2}$ is $\frac{1}{2}\left[n_{1}(s+\right.$ $\left.\left.2 \beta_{1}\right)+r\left(\beta_{1}-\alpha_{1}\right)\right]$. Hence, the sparing number of $G_{1}+G_{2}$ is $\frac{1}{2}\left[\min \left\{\left(n_{1}\left(r+2 \beta_{2}\right)+s\left(\beta_{2}-\alpha_{2}\right)\right),\left(n_{1}\left(s+2 \beta_{1}\right)+r\left(\beta_{1}-\right.\right.\right.\right.$ $\left.\left.\left.\left.\alpha_{1}\right)\right)\right\}\right]$.

In the coming discussion, we discuss the sparing number of certain graph classes using the above proved results.

THEOREM 16. The sparing number of a wheel graph $W_{n+1}=$ $C_{n}+K_{1}$ is

$$
\varphi\left(W_{n+1}\right)= \begin{cases}\frac{n}{2} & \text { if } n \text { is even } \\ \frac{n+3}{2} & \text { if } n \text { is odd }\end{cases}
$$

Proof. A wheel graph $W_{n+1}$ is the graph $C_{n}+K_{1}$ with $2 n$ vertices. The independence set containing maximal incidences con- tains $\left\lfloor\frac{n}{2}\right\rfloor$ vertices, the degree of each of which is 3 . Therefore, the sparing number of $W_{n+1}$ is

$$
\varphi\left(W_{n+1}\right)=2 n-3\left\lfloor\frac{n}{2}\right\rfloor= \begin{cases}\frac{n}{2} & \text { if } n \text { is even } \\ \frac{n+3}{2} & \text { if } n \text { is odd }\end{cases}
$$

This completes the proof.

A helm graph, denoted by $H_{n}$, is the graph obtained by attaching pending a pendant edge to all the vertices of $W_{n+1}$, except its central vertex. Then, we have

THEOREM 17. For a helm graph $H_{n}, \varphi\left(H_{n}\right)=$ $\begin{cases}\frac{n}{2} & \text { if } n \text { is even } \\ \frac{n+3}{2} & \text { if } n \text { is odd. }\end{cases}$

Proof. Note that the size of a helm graph $H_{n}$ is $2 n$. The independence set containing maximal incidences contains $n$ vertices including $\left\lfloor\frac{n}{2}\right\rfloor$ independent vertices of the wheel graph $W_{n+1}$ each of which have degree 4 and $\left\lceil\frac{n}{2}\right\rceil$ pendant vertices. Therefore, the sparing number of $H_{n}$ is given by

$$
\varphi\left(H_{n}\right)=3 n-\left[4\left\lfloor\frac{n}{2}\right\rfloor+\left\lceil\frac{n}{2}\right\rceil\right]= \begin{cases}\frac{n}{2} & \text { if } n \text { is even } \\ \frac{n+3}{2} & \text { if } n \text { is odd. }\end{cases}
$$

This completes the proof.

A closed helm graph, denoted by $\mathrm{CH}_{n}$, is the graph obtained by drawing edges between the pendant vertices of a helm graph. Then,

THEOREM 18. For a closed helm graph $H_{n}, \varphi\left(C_{n}\right)=$ $\begin{cases}\frac{n}{2} & \text { if } n \text { is even } \\ \frac{n+7}{2} & \text { if } n \text { is odd. }\end{cases}$

PROOF. The number of edges in a closed helm graph is $4 n$. Therefore, an independence set having maximal incidences in $C H_{n}$ contains $\left\lfloor\frac{n}{2}\right\rfloor$ vertices in the inner cycle, each of which is of degree 4 and $\left\lfloor\frac{n}{2}\right\rfloor$ vertices in the outer cycle of $C H_{n}$, each of which is of order 3. Therefore, the total edges of $G$ having non-singleton set-labels in $\mathrm{CH}_{n}$ is $7\left\lfloor\frac{n}{2}\right\rfloor$. Therefore,

$$
\varphi\left(C H_{n}\right)=4 n-7\left\lfloor\frac{n}{2}\right\rfloor= \begin{cases}\frac{n}{2} & \text { if } n \text { is even } \\ \frac{n+7}{2} & \text { if } n \text { is odd. }\end{cases}
$$

This completes the proof.

A web graph,denoted by $W(n)$, is a graph obtained by attaching pendant edges to the vertices of the outer cycle of a closed helm graph. Then, we have the following theorem.

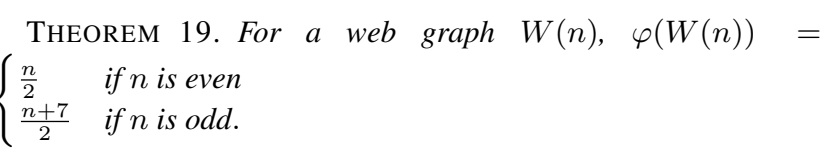

Proof. The number of edges in a web graph $W(n)$ is $5 n$. An independence set having maximal incidence in $G$ consists of $\left\lfloor\frac{n}{2}\right\rfloor$ vertices having degree 4 each from the inner and outer cycles of $W(n)$ and $\left\lceil\frac{n}{2}\right\rfloor$ pendant vertices. Then, the sparing number of the wheel graph is given by

$$
\varphi(W(n))=5 n-\left[7\left\lfloor\frac{n}{2}\right\rfloor+\left\lceil\frac{n}{2}\right\rfloor\right]= \begin{cases}\frac{n}{2} & \text { if } n \text { is even } \\ \frac{n+7}{2} & \text { if } n \text { is odd }\end{cases}
$$

This completes the proof. 


\section{CONCLUSION}

In this paper, we have discussed the admissibility of weak integer additive set-labelings by certain graphs and graph operations and determined their corresponding sparing numbers. Some problems in this area are still open. Finding the sparing number of certain products of arbitrary graphs, certain graph classes with specific properties etc. are some of the promising problems in this area. Problems regarding the admissibility of integer additive setlabelings, both uniform and non-uniform, by certain graphs are also open.

\section{REFERENCES}

[1] K. Abhishek, New directions in the Theory of Set-Valuations of Graphs, Ph.D Thesis, Kannur University, India., 2009.

[2] J. A. Bondy and U. S. R. Murty, Graph Theory, Springer, 2008.

[3] J. A. Gallian, A Dynamic Survey of Graph Labelling, The Electronic Journal of Combinatorics (DS-6), 2014.

[4] K. A. Germina and T. M. K. Anandavally, Integer Additive Set-Indexers of a Graph: Sum Square Graphs, Journal of Combinatorics, Information and System Sciences, 37(24)(2012), 345-358., DOI: 10.12988/imf.2013.310188.

[5] K. A. Germina and N. K. Sudev, On Weakly Uniform Integer Additive Set-Indexers of Graphs, International Mathematical Forum, 8(37)(2013), 1827-1834.

[6] F. Harary, Graph Theory, Addison-Wesley Publishing Company Inc., 1969.

[7] M. B. Nathanson, Additive Number Theory, Inverse Problems and Geometry of Sumsets, Springer, 1996.
[8] N. K. Sudev and K. A. Germina, On Integer Additive SetIndexers of Graphs, International Journal of Mathematical Sciences \& Engineering Applications, 8(2)(2014), 11-22.

[9] N. K. Sudev and K. A. Germina, A Characterisation of Weak Integer Additive Set-Indexers of Graphs, Journal of Fuzzy Set Valued Analysis, 2014(2014), 1-7., DOI:10.5899/2014/jfsva00189.

[10] N. K. Sudev and K. A. Germina, A Note on Sparing Number of Graphs, Advances and Applications in Discrete Mathematics, 14(1)(2014), 51-65.

[11] N. K. Sudev and K. A. Germina, On Weak Integer Additive Set-Indexers of Certain Graph Classes, Journal of Discrete Mathematical Sciences and Cryptography, 18(1-2)(2015), 117128., DOI : 10.1080/09720529.2014.962866

[12] N. K. Sudev and K. A. Germina, Weak Integer Additive SetIndexers of Graph Operations, Global Journal of Mathematical Sciences: Theory and Practical, 6(1)(2014), 25-36.

[13] N. K. Sudev and K. A. Germina, Weak Integer Additive SetIndexers of Graph Operations, TechS Vidya E-Journal of Research, 2(2013-14), 28-38.

[14] N. K. Sudev, K. A. Germina and K. P. Chithra, Weak Set-Labeling Number of Certain Integer Additive SetLabeled Graphs, International of Computer Applications, 114(2)(2015), 1-6., DOI: 10.5120/19947-1772.

[15] N. K. Sudev, K. A. Germina and K. P. Chithra, Weak Integer Additive Set-Labeled graphs: A Creative Review, Asian European Journal of Mathematics, 8(3)(2015), 1-22., DOI: $10.1142 / \mathrm{S} 1793557115500527$.

[16] D. B. West, Introduction to Graph Theory, Pearson Education Inc., 2001. 